\title{
KCNMA1 cooperating with PTK2 is a novel tumor suppressor in gastric cancer and is associated with disease outcome
}

Gaoxiang Ma ${ }^{1,2 \dagger}$, Hanting Liu ${ }^{1,2 \dagger}$, Qiuhan Hua ${ }^{1,2+}$, Meilin Wang ${ }^{1,2}$, Mulong Du ${ }^{1,2}$, Yadi Lin ${ }^{1,2}$, Yuqiu Ge $e^{1,2}$, Weida Gong ${ }^{3}$, Qinghong Zhao ${ }^{4}$, Fulin Qiang ${ }^{5}$, Guoquan Tao ${ }^{6}$, Zhengdong Zhang ${ }^{1,2,7^{*}}$ and Haiyan Chu ${ }^{1,2,7^{*}}$

\begin{abstract}
Background: Inactivation of tumor suppressor genes by promoter hypermethylation plays a key role in the tumorgenesis. It is necessary to uncover the detailed pattern of whole genome-wide abnormal DNA methylation during the development of gastric cancer (GC).

Method: We performed a genome-wide methylation detection using 12 paired of GC tissues and their corresponding normal tissues. Methylation-specific PCR (MSP) and bisulphite sequencing (BSP) were used to measure methylation status of specific CpG site. Based on the bioinformatic analysis, the cell phenotypes and mouse model experiments were constructed to detect effect of the target gene. Using the Kaplan-Meier survival curve, the clinical value of KCNMA1 was assessed in GC patients.

Results: The CpG site cg24113782 located at the promoter of KCNMA1 showed the most significant difference, contributing to the commonly silenced KCNMA1in gastric cancer cells and primary GC tissues. The promoter methylation of KCNMA1 was detected in $68.7 \%$ (77/112) of tumor tissues, compared with $16.2 \%(18 / 112)$ of normal tissues $(P<0.001)$. The survival curve indicated that KCNMA1 hypermethylation was significantly associated with the shortened survival in $G C$ patients $(P=0.036)$. KCNMA1 significantly inhibited biological malignant behavior of gastric cancer cell by inducing cell apoptosis in vitro, and suppressed xenograft tumor growth in subcutaneous mouse models (both $P<0.001$ ). Furthermore, the anti-tumor effect of KCNMA1was mediated through suppressing the expression of PTK2.
\end{abstract}

Conclusion: KCNMA1 is a critical tumor suppressor in gastric carcinogenesis and its hypermethylation is an independent prognostic factor in patients with gastric cancer.

Keywords: Gastric cancer, KCNMA1 Methylation, Prognosis

\section{Background}

Gastric cancer (GC) is one of the most common malignancies and remains the second leading cause of cancerrelated death worldwide. Despite modified surgical and adjuvant treatment strategy, the prognosis of GC patients is poor, with a 5-year overall survival of less than $25 \%[1,2]$. There are considerable evidences indicating that epigenetic alterations, particularly

\footnotetext{
*Correspondence: drzdzhang@gmail.com; chy_grape@126.com

${ }^{\dagger}$ Equal contributors

'Department of Environmental Genomics, Jiangsu Key Laboratory of Cancer Biomarkers, Prevention and Treatment, Cancer Center, Nanjing Medical University, Nanjing, China

Full list of author information is available at the end of the article
}

inactivation of tumor suppressor genes through promoter hypermethylation, play an important role in the development and progression of GC [3]. Identification of such novel genes targeted by promoter hypermethylation may provide insights into alternative approaches for diagnostic and therapeutic targets and the epigenetic mechanisms in GC. In normal cells, the pattern of DNA methylation is handed down to the daughter cells during mature cell division. However, the aberrant alterations in the DNA methylation profile of mature cells are frequently observed in many human cancers, including GC $[4,5]$. Therefore, identification of the differences of the DNA methylation status in GC to reveal the role of 
epigenetic instability on the initiation and progression of GC is necessary.

To uncover the genome-wide DNA methylation profiles of GC in a more comprehensive way, we performed a microarray analysis between gastric cancer issues and their matched normal tissues with Illumina Infinium Human Methylation450 BeadChip array that include $>485,000 \mathrm{CpG}$ sites distributed throughout the genome [6]. We found that the gene, potassium channel, calcium activated large conductance subfamily $\mathrm{M}$ alpha, member 1(KCNMA1), the function of which remains largely unexplored, was moderated by promoter methylation in gastric cancer. KCNMA1 (also named BK) potassium channels are a diverse class of ion channels expressed in many different cell types [7]. The protein encoded by KCNMA1 represents the voltage and $\mathrm{Ca}^{2+}$-activated $\mathrm{K}^{+}$ channel, and is involved in the feedback inhibition of the action potential frequency and $\mathrm{Ca}^{2+}$ influx $[8,9]$. Emerging evidences have identified that the $\mathrm{Ca}^{2+}$ is closely related to cell apoptosis $[10,11]$. Moreover, by bioinformatics analysis based on The Cancer Genome Atlas (TCGA), we found the KCNMA1could regulate the expression of $F A K$ (focal adhesion kinase), also named PTK2, which is a non-receptor tyrosine kinase and moderate cancer proliferation, migration and survival [12]. And it may regulate the cell apoptosis by PI3K-AKT pathway [13]. It is possible that the $\mathrm{Ca}^{2+}$ is involved in apoptosis by cooperating with PTK2.

We reasoned the KCNMA1 contribute to the $\mathrm{GC}$ risk by regulating the key apoptosis gene PTK2. In this study, therefore, we set out to explore the expression profile, epigenetic regulation, biological function, molecular basis and clinical application of KCNMA1 in GC.

\section{Methods}

\section{GC cell lines}

A total of four GC cell lines (i.e., MGC-803, BGC-823, SGC-7901, and MKN-28) and one normal human gastric epithelial cell (GES-1) were used in this study. All cell lines were maintained in RPMI-1640 medium (Gibco BRL, Rockville, Maryland, USA) with 10\% fetal bovine serum (Gibco BRL). And the identity of the cell lines were confirmed by short tandem repeat (STR).

\section{Gastric tissue samples}

Seventy-nine paired tumor and adjacent non-tumor gastric samples were obtained from GC patients at the Second Affiliated Hospital of Nanjing medical University in Nanjing, China. A total of 75 patients with histologically-confirmed gastric cancer and adjacent non-tumor tissues were evaluated for KCNMA1 with real-time PCR (RT-PCR) and 112 patients with methylation-specific PCR (MSP). The 75 paired of GC tissues were mainly collected from The Second Affiliated
Hospital of Nanjing Medical University, and 112 GC tissues were from the First Affiliated Hospital of Nanjing Medical University without paired adjacent tissues. All subjects of this study signed informed consent for obtaining the study specimens.

\section{Genome-wide Methylation Profiling}

DNA methylation analysis was performed by Shanghai Genergy Co. Ltd (Shanghai, China) using the Illumina Human Methylation450 BeadChip (Illumina). These arrays contain probes for approximately 450,000 CpG loci sites. Target was prepared and hybridized according to the "Illumina Infinium HD Methylation Assay, Manual Protocol". The methylation level was computed as a $\beta$ value according to the normalized probe fluorescence intensity ratios between methylated and unmethylated signals: $\beta$ value $=$ signal intensity of the methylated allele (sum of signal intensity of the unmethylated and methylated allele + 100). The DNA methylation level for each interrogated CpG site was evaluated as a $\beta$ value, which ranged from 0 (not methylated) to 1 (fully methylated). The significant $P$ values of the normal tissue and tumor tissue groups were calculated by paired Wilcox non parametric test, and the Benjamini and Hochberg method were used to carry out multiple test correction calculation FDR [14]. We chose the maximum difference of $\beta$ value between the normal tissue and tumor tissue groups in further research.

\section{RNA extraction and Quantitative real-time PCR (qRT PCR)}

The total RNA was extracted from tissues using Trizol reagent (Invitrogen, CA, USA). The cDNA was synthesized using M-MLV reverse transcriptase (Invitrogen) after RNA extraction according to the manufacturer's instruction. The expression level of genes was detected by qRT-PCR using SYBR Green assays (TaKaRa Biotechnology, Dalian, China). Glyceraldehyde 3-phosphate dehydrogenase $(G A P D H)$ was chose to act as an internal control, and the assay was conducted by ABI 7900 system (Applied Biosystems, CA, USA). To evaluate the primer efficiency, we have used the standard curve to calculate the amplification efficiency. The amplification efficiency of GAPDH, KCNMA1 and PTK2 was 98.1, 96.3 and $97.5 \%$ respectively. The expression of each gene was quantified according to fold change using $2^{-\Delta \Delta C t}$ methods. The primers sequences are available in Additional file 1: Table S1.

\section{DNA extraction, MSP and BSP}

The DNA of tissues was obtained using E.Z.N.A ${ }^{\text {ma }}$ tissue DNA kit (Omega Bio-Tek. USA). Then the tissue DNA was modified by EZ DNA Methylation-Gold ${ }^{\mathrm{mw}}$ Kit (Zymo Research) according to the manufacturer's instruction. The MSP and BSP primer was designed by the Methyl 
Primer Express v1.0 (Applied Biosystems), as shown in Additional file 1: Table S1.

\section{Construction of KCNMA1 expression plasmid and RNA interference}

The full-length open reading frame sequence of KCNMA1 was constructed by GenScript USA Inc. (Nanjing, China) and then was subcloned into the mammalian expression vector pIRES-EGFP. The product was verified by DNA sequencing. Three small interfering RNA (siRNA) were synthesized to target PTK2 (RiboBio, Guangzhou, China). After detection of the interference efficiency, si-PTK2-2 (named si-PTK2 in this study) had the optimal efficiency and was selected for the following study. The sequences are shown in Additional file 1: Table S1. GC cells, MGC-803 and BGC-823, were transiently transfected with the KCNMA1 over-expression plasmid and si-PTK2 using Lipofectamine 2000 (Invitrogen, Carlsbad, CA, USA) transfection reagent according to the instruction. The pIRES-EGFP empty vector was used as negative control (NC).

\section{The malignant behaviors of cancer cells}

Using GC cells, we performed a series of assays to detect the effects of KCNMA1 on the malignant behaviors including apoptosis assay, proliferation, colony formation and migration. The detail of assay conditions was shown in Additional file 2.

\section{Subcutaneous xenograft models in vivo}

MGC803 cells $\left(1 \times 10^{7}\right.$ cells in $0.2 \mathrm{ml}$ PBS $)$ that was stably transfected with KCNMA1 expression vector or empty vector were subcutaneously injected into the dorsal right flank of 5-week-old male Balb/c nude mice $(n=10$ per group). The tumor diameter in the nude mice was measured every 2 days for $2-3$ weeks. After 20 days, all mice were sacrificed and the tumor weight and size were measured. The experiment was approved by the Animal Ethics Committee of Nanjing Medical University.

\section{Statistical analysis}

The independent or paired $t$ test was used to calculate the difference between two preselected groups or paired samples. The associations between the KCNMA1 methylation and expression and clinic opathological characteristics of GC patients were compared using Pearson's $\chi^{2}$ test. The Kaplan Meier survival curves and log-rank test were used to evaluate the relation between the overall survival and methylation status. The $P<0.05$ was regarded as statistical significance.

\section{Results}

Identification of methylation status between gastric cancer tissues and normal tissues

Twelve paired of the tumor and the paired normal tissues were profiled (Additional file 1: Table S1). Results of hierarchical clustering analysis on the most significantly hypermethylation CpG site are shown in Fig. 1a. This analysis revealed a remarkable segregation between the tumor and the paired normal tissues. Through further analysis, we found that the most of top 100 hypermethylation site locate the promoter of the genes (Fig. 1b). And the top 10 high methylated CpG sites can well distinguish the tumor tissues from the normal tissues (Fig. 1c). Interesting, the CpG site cg24113782 with most significant difference was located in the promoter region of KCNMA1. Moreover, this result was also supported by the data from the independent TCGA data. The above results indicated cg24113782 had a notably high $\beta$-score value in the cancer tissues compared normal tissues $(P<0.001)$ (Additional file 3: Figure S1). In addition, this finding was also identified in the Human Methylation 27 array from TCGA, which has a low density and mainly focuses on CpG-sites mapping to gene promoter regions. Although the cg24113782 site was not included in the HumanMethylation 27 array, we found the other CpG site cg04688368 in the HumanMethylation27 array which also located on the KCNMA1 promoter region. The $\beta$-score value of $\operatorname{cg} 04688368$ between tumor and paired normal tissues had a significant difference in the paired GC tissue $(P<0.001$, Additional file 3: Figure S1).

\section{Silence or downregulation of KCNMA1 by promoter methylation in gastric cancer cells and tissues}

The expression of KCNMA1 was detected in the GC cells (i.e., MGC-803, BGC-823, MKN-82, SGC-7901) and the normal human gastric epithelial cell line (GES1) using RT-PCR (Additional file 3: Figure S2). The mRNA expression of KCNMA1 was silenced or reduced in the GC cells compared with normal human gastric epithelial cell. To identify whether the cancer cell methylation directly mediates KCNMA1 expression, we treated the two cell lines (i.e., MGC-803 and BGC-823) with the demethylation agent, 5-Aza-2'-deoxycytidine (5-Aza; Sigma-Aldrich), for $72 \mathrm{~h}$. Notably, this treatment restored expression of KCNMA1 in the two silenced cell lines (Additional file 3: Figure S2), suggesting that the expression silence of KCNMA1 was moderated by the aberrant promoter methylation.

To detect the contribution of promoter methylation to the down-regulation of KCNMA1 for tumor and paired normal tissues, methylation status of its promoter was examined by methylation-specific PCR (MSP) in 112 paired tissues. We found 68.7\% (77/112) GC tissues were 

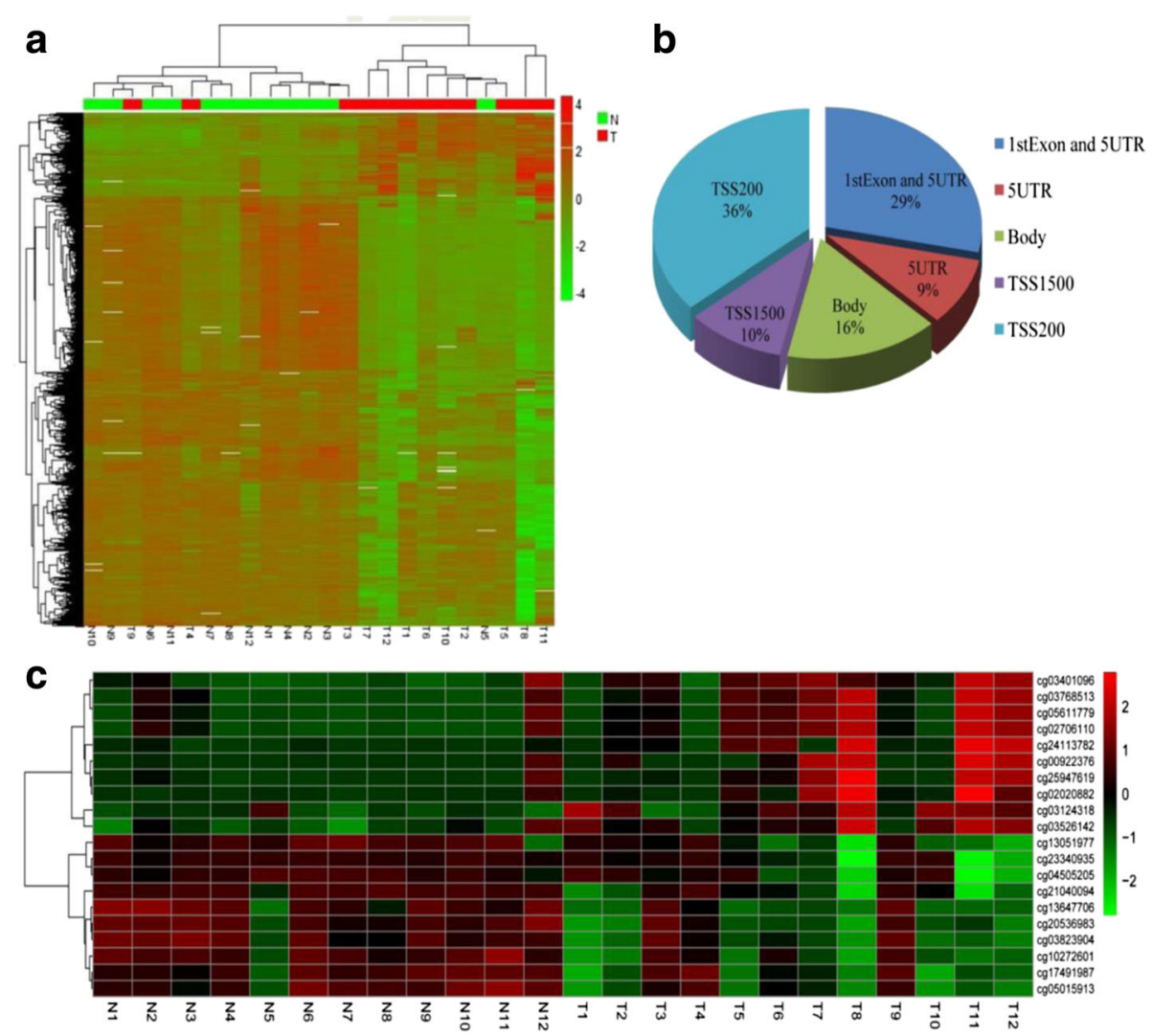

Fig. 1 Hierarchical clustering analysis of the microarray assay. a The heat map of the different methylated site between the gastric carcinoma and paired corresponding normal tissues. b The gene location of the most 100 significantly hypermethylated $\mathrm{CpG}$ sites. c Hierarchical clustering analysis on the most 10 significantly hypermethylated and hypomethylationCpG sites. N, normal tissues, T, gastric carcinoma tissues. TSS1500, 1500 bases before the transcription start site. TSS200, 200 bases before the transcription start site. Body, the intron and exon of gene

methylated, but only $16.2 \%(18 / 112)$ normal tissues were methylated (Fig. 2a), and the BSP results also confirmed this finding (Fig. 2b). In addition, we detected the expression of KCNMA1 in 75 paired of cancer and normal tissues. The expression level of KCNMA1 in cancer tissues was significantly decreased compared with normal tissues $(P=0.008$, Fig. 2c, d). The same result was found in TCGA and GEO data (Additional file 3: Figure S1). As shown in Table 1, the aberrant KCNMA1 methylation status in GC tissues was associated with tumor sizes and depth of invasion. Meanwhile, we found the aberrant expression contributed to the tumor sizes in Table 2.

\section{KCNMA1 is an independent predictor of prognosis in patients with GC}

The association between KCNMA1 methylation status and clinical outcome was analyzed in 91 patients with GC with known survival data. As shown in Fig. 2e, GC patients with KCNMA1 methylation had significantly shorter survival than others $(P=0.038, \log$-rank test).

\section{Ectopic expression of KCNMA1 suppressed GC cell} proliferation, migration, invasion and colony formation Considering frequent silencing of KCNMA1 in primary cancers and GC cell lines but not in normal gastric tissues, it suggested that KCNMA1 may act as probably a tumor suppressor. KCNMA1-expressing plasmid was stably transfected into MGC803 and BGC823 cells. Reexpression of KCNMA1 was confirmed by RT-PCR and Western blot analysis (Fig. 3a). Firstly, CCK- 8 assay showed that proliferation of MGC803 and BGC823 cells were remarkably suppressed after KCNMA1overexpression for $24 \mathrm{~h}, 48 \mathrm{~h}$ and $72 \mathrm{~h}$ compared with those transfected with $\mathrm{NC}$ vectors (Fig. 3b). Compared with MGC803 and BGC823 cells transfected with NC vector, the cells with over-expression of KCNMA1 for $48 \mathrm{~h}$ showed significantly decreased migration ability $(P<0.01$, Fig. $3 c)$. Besides, the suppression effect on invasion was also observed in both the two cells after $48 \mathrm{~h}$ of transfection $(P<0.01$, Fig. 3d). Moreover, the inhibitory effect on GC cell growth was further confirmed by colony formation assay. The colonies formed by KCNMA1-transfected 


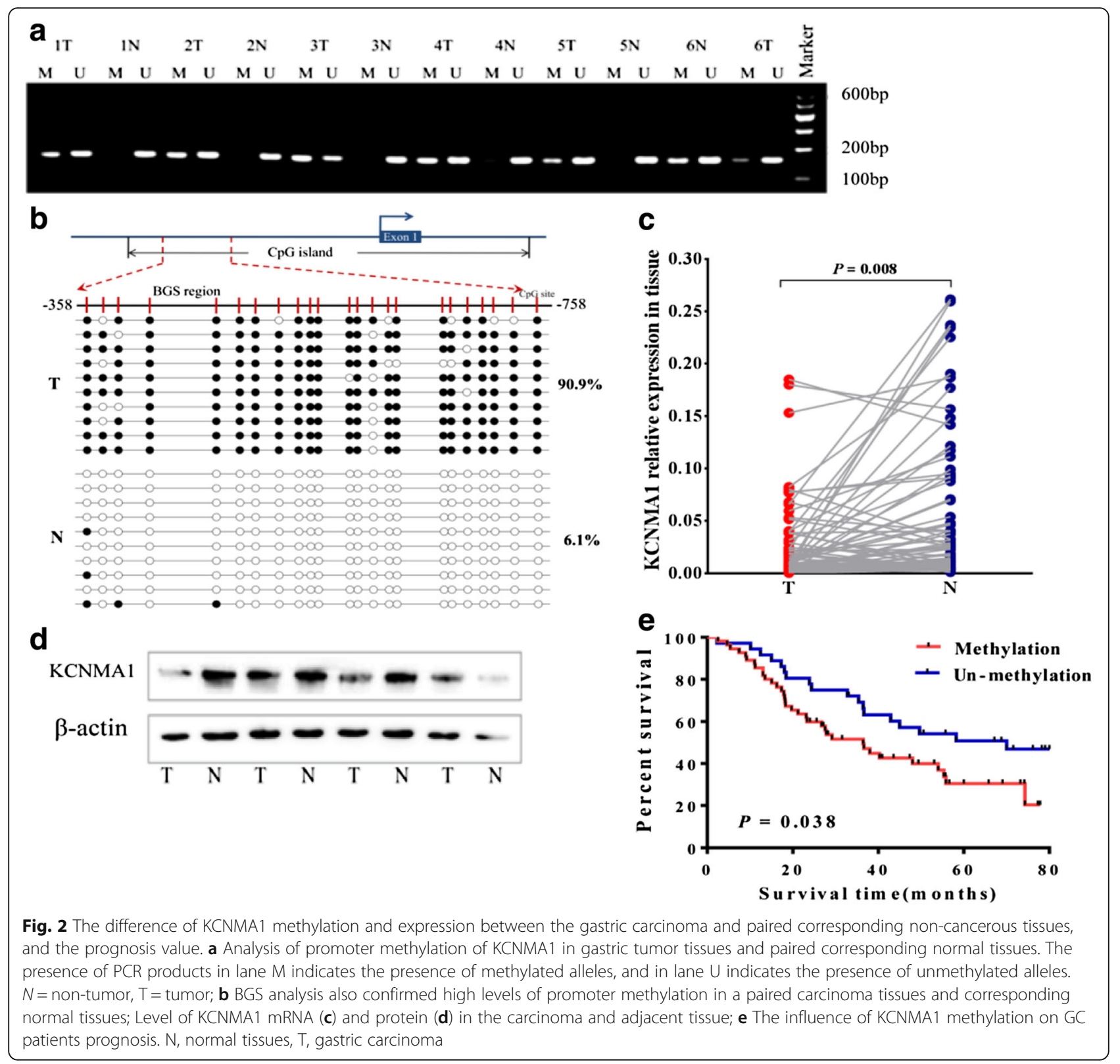

cells were significantly smaller and fewer than those formed by NC vector-transfected cells $\quad(P<0.01$, Fig. 3e).

\section{KCNMA1 induced cell apoptosis}

Suppression of tumor cell growth is usually involved in concomitant activation of cell apoptosis pathways. Therefore we detected the contribution of apoptosis to the growth inhibition of KCNMAlover-expression cells using flow cytometry (Fig. 4f). The results indicated an increase in the numbers of both early apoptotic cells $(P<0.01)$ and late apoptotic cells $(P<0.01)$ in KCNMA1transfected MGC803 and BGC823 cells compared with those transfected with NC vector (Fig. 4f).

\section{Identification of genes modulated by KCNMA1 in GC cell} lines

To gain insights into the molecular basis of apoptosis KCNMA1-modulated, the downstream target genes were characterized through cBioPortal for Cancer Genomics (Additional file 3: Figure S3) and found that the PTK2 gene involved in FAK apoptosis pathways may be correlated with KCNMA1. Firstly, we found that the PTK2 was significantly high expression in tumor tissues than paired normal tissues (Fig. 4a). Then, the correlation between the KCNMA1 and PTK2 was examined in gastric cancer tissues, and the result indicated that the expression levels of KCNMA1 and PTK2 were significantly correlated in a negative direction $(r=-0.364, P<0.01$, 
Table 1 Clinicopathological features of KCNMA1 promoter methylation in 112 patients with GC

\begin{tabular}{|c|c|c|c|}
\hline Factors & $\begin{array}{l}\text { Methylated } \\
(N=77)\end{array}$ & $\begin{array}{l}\text { Non-methylated } \\
(N=35)\end{array}$ & $P$ value \\
\hline Age $($ mean $\pm S D)$ & $64.44 \pm 1.00$ & $61.81 \pm 1.86$ & 0.182 \\
\hline \multicolumn{4}{|l|}{ Gender } \\
\hline Male & 50 & 22 & \multirow[t]{2}{*}{0.879} \\
\hline Female & 27 & 13 & \\
\hline \multicolumn{4}{|l|}{ Tumor sizes } \\
\hline$\leq 5 \mathrm{~cm}$ & 35 & 26 & \multirow[t]{2}{*}{0.005} \\
\hline$>5 \mathrm{~cm}$ & 42 & 9 & \\
\hline \multicolumn{4}{|l|}{ Depth of invasion } \\
\hline $\mathrm{T} 1+\mathrm{T} 2$ & 17 & 2 & \multirow[t]{2}{*}{0.032} \\
\hline $\mathrm{T} 3+\mathrm{T} 4$ & 60 & 33 & \\
\hline \multicolumn{4}{|c|}{ Lymphnode metastasis } \\
\hline No & 22 & 7 & \multirow[t]{4}{*}{0.808} \\
\hline N1 & 23 & 12 & \\
\hline N2 & 17 & 9 & \\
\hline N3 & 15 & 7 & \\
\hline \multicolumn{4}{|l|}{ Metastasis } \\
\hline Mo & 67 & 29 & \multirow[t]{2}{*}{0.560} \\
\hline M1 & 10 & 6 & \\
\hline \multicolumn{4}{|l|}{ TNM stages } \\
\hline । & 9 & 2 & \multirow[t]{4}{*}{0.687} \\
\hline$\|$ & 19 & 11 & \\
\hline III & 38 & 16 & \\
\hline IV & 11 & 6 & \\
\hline
\end{tabular}

The entries in bold showed the $P$ value is less than 0.05

Fig. 4b), which was further confirmed by the GEO data ( $r$ $=-0.25, P=0.036$, Fig. 4b) (GSE29272). Taking into consideration the published researches, the function of antitumor of PTK2 was also found in this study (Fig. 4e, f, $g$ and Additional file 3: Figure S5). When KCNMA1-expressing plasmid was transfected into MGC803 and BGC823 cells, the expression level of PTK2 was detected by RT-PCR and western blotting. As shown in Fig. 4d, the expression of PTK2 had a significant decrease compared with NC cells.

\section{Knockdown of PTK2 expression by siRNA}

In order to identify whether the observed antitumor effects of KCNMA1 was the consequence of its downregulation of PTK2 gene, knockdown of PTK2 expression was achieved by siRNA interference. RT-PCR results of the interfered cells indicated that PTK2 expression was remarkable decrease, except for si-PTK2-1 (Additional file 3: Figure S4). In the further study, si-PTK2 with the highest inhibition ratio up to $75 \%$ was selected. Malignant phenotypes of MGC803 and BGC823 cells
Table 2 The relationship between KCNMA1 expression and clinicopathological feature of 75 GC patients

\begin{tabular}{|c|c|c|c|c|}
\hline \multirow{2}{*}{$\begin{array}{l}\text { Clinicopatholocical } \\
\text { variables }\end{array}$} & \multirow{2}{*}{$\begin{array}{l}\text { Number of } \\
\text { each group }\end{array}$} & \multicolumn{2}{|c|}{ KCNMA1 expression } & \multirow[t]{2}{*}{$P$ value } \\
\hline & & High & Low & \\
\hline \multicolumn{5}{|l|}{ Age(years) } \\
\hline$<60$ & 27 & 13 & 14 & \multirow[t]{2}{*}{0.878} \\
\hline$\geq 60$ & 48 & 24 & 24 & \\
\hline \multicolumn{5}{|l|}{ Sex } \\
\hline Male & 62 & 30 & 32 & \multirow[t]{2}{*}{0.720} \\
\hline Female & 13 & 7 & 6 & \\
\hline \multicolumn{5}{|l|}{ Tumor size } \\
\hline$\leq 5 \mathrm{~cm}$ & 42 & 26 & 16 & \multirow[t]{2}{*}{0.014} \\
\hline$>5 \mathrm{~cm}$ & 33 & 11 & 22 & \\
\hline \multicolumn{5}{|l|}{ Tumor site } \\
\hline Cardia & 26 & 12 & 14 & \multirow[t]{2}{*}{0.922} \\
\hline Non-cardia & 47 & 24 & 23 & \\
\hline \multicolumn{5}{|l|}{ Histological type } \\
\hline Diffuse & 41 & 21 & 20 & \multirow[t]{2}{*}{0.721} \\
\hline Intestinal & 34 & 16 & 18 & \\
\hline \multicolumn{5}{|l|}{ Depth of invasion } \\
\hline $\mathrm{T} 1+\mathrm{T} 2$ & 15 & 4 & 11 & \multirow[t]{2}{*}{0.179} \\
\hline $\mathrm{T} 3+\mathrm{T} 4$ & 60 & 20 & 35 & \\
\hline \multicolumn{5}{|c|}{ Lymph nodedistant metastasis } \\
\hline $\mathrm{N} 0+\mathrm{N} 1$ & 20 & 11 & 9 & \multirow[t]{2}{*}{0.554} \\
\hline $\mathrm{N} 2+\mathrm{N} 3$ & 55 & 26 & 29 & \\
\hline \multicolumn{5}{|l|}{ Distant metastasis } \\
\hline Mo & 62 & 30 & 32 & \multirow[t]{2}{*}{0.720} \\
\hline $\mathrm{M} 1$ & 13 & 7 & 6 & \\
\hline \multicolumn{5}{|l|}{ TNM } \\
\hline $\mid+\|$ & 22 & 10 & 12 & \multirow[t]{2}{*}{0.665} \\
\hline$I I I+I V$ & 53 & 27 & 26 & \\
\hline
\end{tabular}

The entries in bold showed the $P$ value is less than 0.05

were monitored repeatedly with both KCNMA1 overexpressing and PTK2 knockdown.

\section{Repeating observation on cell phenotype after KCNMA1- expressing plasmid and si-PTK2 transfected}

In the repeated CCK- 8 assay, we found that suppressed role of KCNMAlon GC cells proliferation was markedly weakened with co-transfection of KCNMA1 vector and si-PTK2. As the presented in Fig. 4e, there was no significant difference in proliferation ratio of treated BGC823 and MGC803cells in the co-transfection of KCNMA1 vector and si-PTK2 groups, compared with only the si-PTK2 groups. Similarly, inhibitory ability on gastric cancer cell migration and invasion was also attenuated by si-PTK2, that is, KCNMA1 did not have the ability to suppress migration and invasion of gastric cancer cells after PTK2 was knockdown 


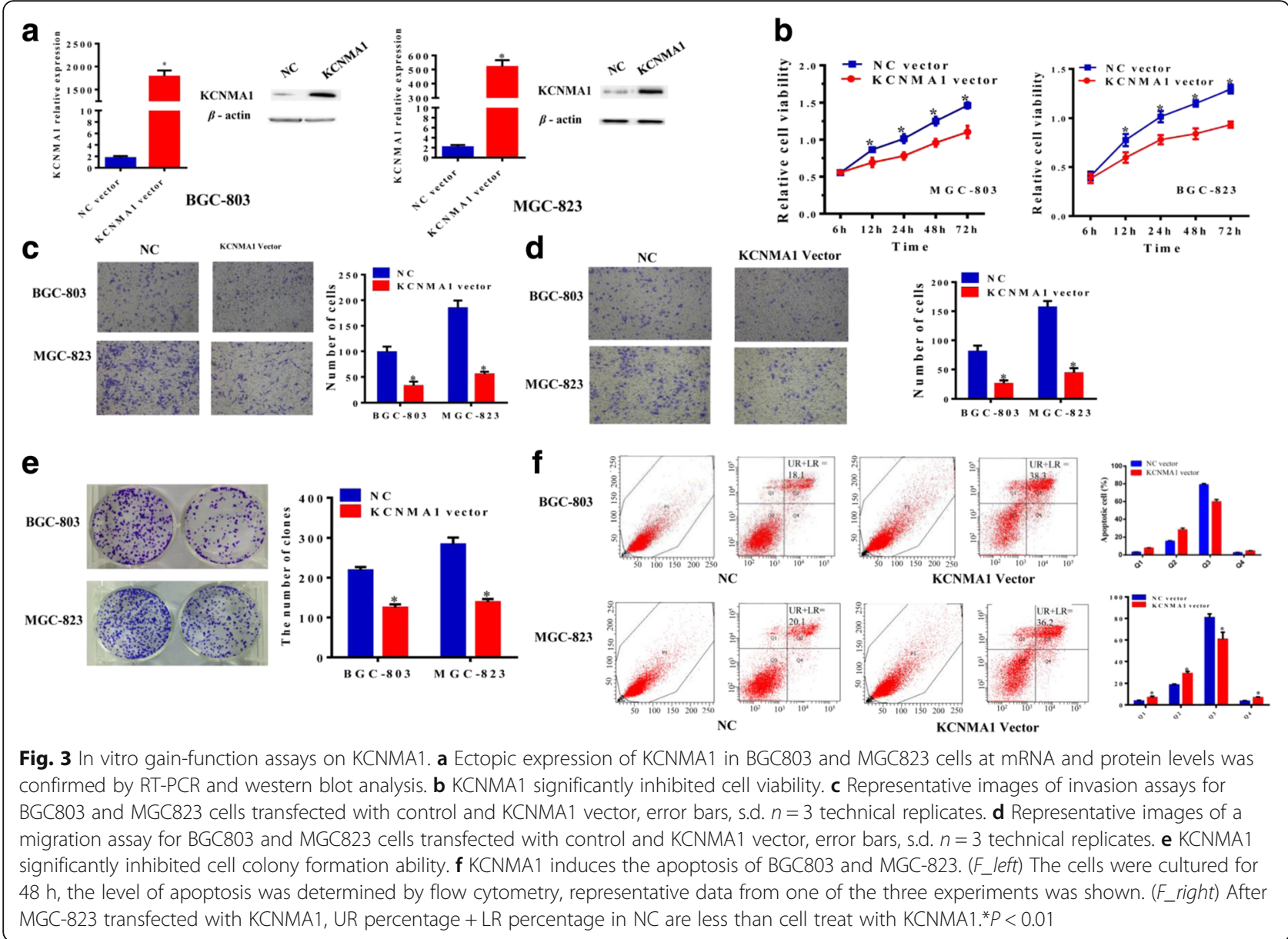

(Fig. 4f). Furthermore, the differences were not found on inhibiting the cell colony formation between KCNMA1 vector and si-PTK2 co-transfected cells and cells with transfection of si-PTK2 groups (Fig. 4g).

\section{KCNMA1 repressed the growth of subcutaneous xenograft} tumours in nude mice

The subcutaneous xenograft tumor models were used to explore the effect of $K C N M A 1$ on gastric tumor cell growth in vivo. The empty vector transfected and subcutaneously injecting KCNMA1-transfected MGC803 cells were inoculated in nude mice. Then the status of subcutaneous tumor growth was recorded and monitored in the two groups. As shown in Fig. 5a, b and d, KCNMAlcan significantly attenuates the growth of tumor volume and tumor volumes were compared with control cells $(P<0.001)$. And compared with $\mathrm{NC}$ cells at termination of the experiment, the weight of tumors with KCNMA1-transfected cells was also significantly reduced $(P<0.001$, Fig. $5 c)$.

\section{Discussion}

In the present study, we have identified that KCNMA1 is commonly silenced or down-regulated in primary gastric cancer tissues and gastric cancer cell lines due to promoter hypermethylation. In addition, the publicly available GEO and TCGA datasets were used to confirm that finding. The expression of KCNMA1can be reactivated by pharmacological demethylation, which inferred that promoter methylation is the primary mechanism for the silencing of KCNMA1 in GC.

The clinical outcome of GC generally depends on the aggressiveness of individual tumors and growth status. TNM stage is still the critical clinical factor that influences the prognosis of cancer patient. However, recurrence of many GC patients often occurs at early stages. Identifying additional prognostic makers, which can provide better risk assessment to extend survival, is necessary and crucial. We explored the clinical importance of KCNMA1 methylation in 91 patients with GC, and found KCNMA1methylation was an independent predictive biomarker of unfavorable outcome in patients with GC by multivariate Cox regression analysis. Many studies have indicated the promoter methylation can serve as a promising prognostic biomarker in gastric cancers [5, 15-17]. Our findings show that KCNMA1 hypermethylation may act as a new valuable marker for 

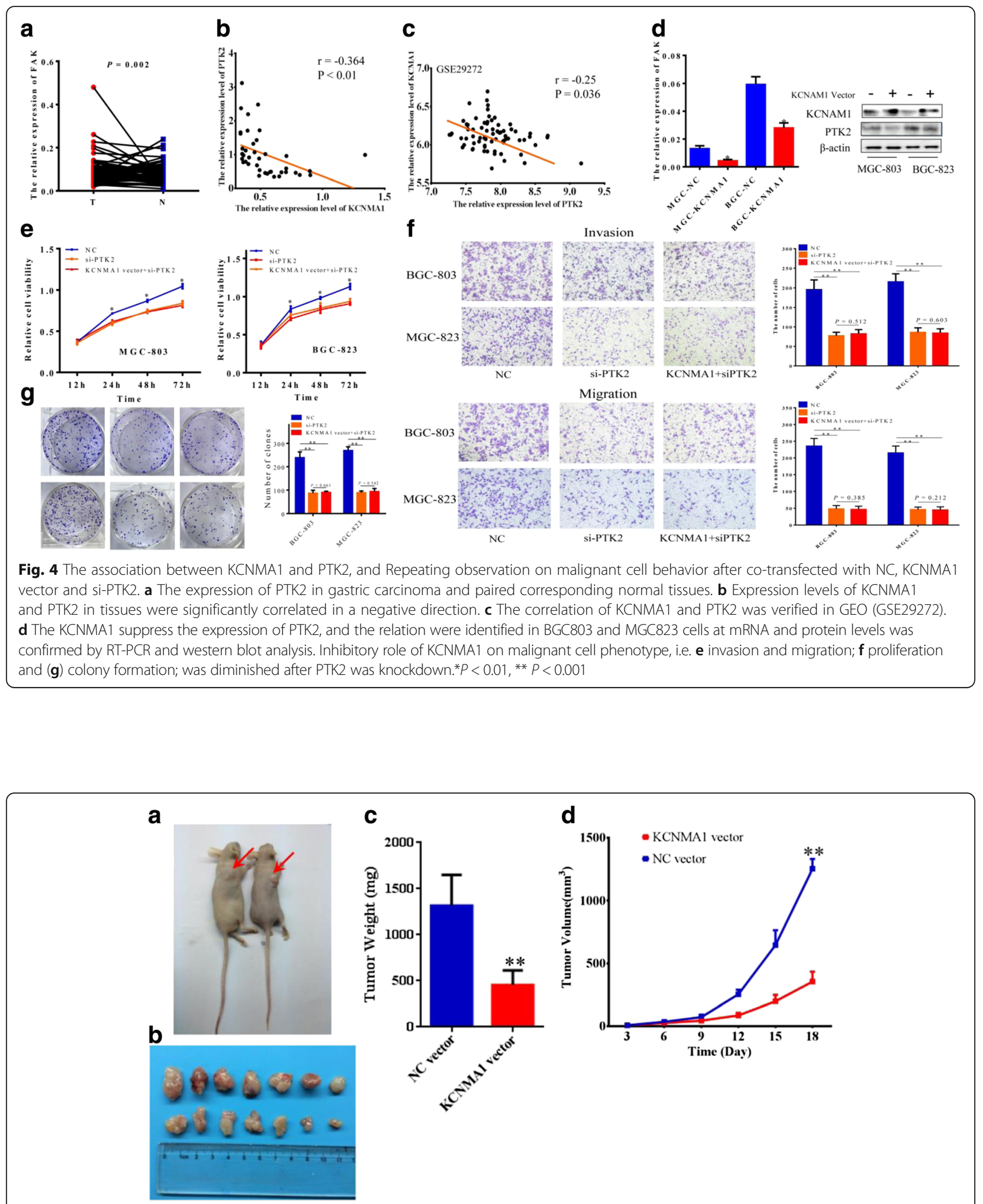

Fig. 5 KCNMA1 suppresses gastric cancer cell growth in xenograft mice. a Representative burdened nude mice in KCNMA1 re-expressed and NC in MGC803 cells. Red arrows show position of subcutaneous tumors. b Representative xenografts in KCNMA1 re-expressed and NC in MGC803 cells. c Tumor weight in nude mice at the 18 day after inoculation of KCNMA1 NC and re-expressed MGC803 cells. Bars: mean of 7 mice. d The tumor volumes for KCNMA1 NC and re-expressed MGC803 cell xenografts. Points: mean of 7 mice. ${ }^{* *} P<0.001$ 
predicting the prognosis of patients with GC. KCNMA1 was uncovered to be commonly downregulated in patients with GC, which implied the key role of the functional silence of KCNMA1 because of promoter methylation during carcinogenesis. In this study, we have not found the difference of methylated KCNMA1 between intestinal and diffuse tumor types, which meant the KCNMA1 may be not involved in the lauren classification.

We further investigated the putative tumor suppressor function of KCNMA1 in human gastric cancer both in vitro and in vivo assays. Compared with empty vector transfection, ectopic expression of KCNMA1 in the down-regulated MGC803 and BGC823 cells significantly suppressed cell viability and reduced colony formation ability. Moreover, MGC803 and BGC823 cells of overexpressing KCNMA1 showed significantly decreased ability in invasion and migration and suppressed the growth of subcutaneous xenograft tumors in nude mice. The mechanism by which KCNMA1 suppressed malignant behaviors of the gastric cancer cell was mediated by inducing cell apoptosis. The apoptosis by KCNMA1 was associated with the focal adhesion kinase (FAK), also named PTK2, which is a cytoplasmic protein tyrosine kinase. PTK2 can enhance tumor progression and metastasis through effects on cancer cells, as well as stromal cells of the tumor microenvironment [18-20]. The kinase-dependent and kinase-independent functions of PTK2 moderate cell movement, invasion, survival and cancer stem cell self-renewal [21]. We found the KCNMA1 down-regulated the expression of PTK2, and promoted the apoptosis of GC cell lines.

The role of PTK2 as a major player in suppressing the apoptosis of cancer cell has been well revealed, and PTK2 is often expressed at aberrant high levels in cancer cells [22-24]. Studies have identified its downstream target PI3K-AKT pathway was involved in the functions of various kinds of cells including apoptosis [13, 25, 26]. Moreover, emerging studies have confirmed the interaction between the KCNMA1 and PI3K [27]. Our research revealed the molecular mechanism that the KCNMA1 can moderate the PTK2. This present study showed the significantly reduced cell proliferation, invasion and metastasis by KCNMA1 were related to induction of apoptosis, in which the PTK2 play a crucial role. We proposed that aberrant KCNMA1 expression can disturb the $\mathrm{K}^{+}$channel function, and thus activate the FAK pathway, which play a key role on the cell apoptosis [21]. However, it needed further functional studies to identify.

Some studies have explored the mechanism of KCNMA1 in the tumorigenesis. KCNMA1 protein (also named BK) was the pore-forming $\alpha$ subunit of the $\alpha$-subunit of the large conductance, voltage and $\mathrm{Ca}^{+}$-activated $\mathrm{K}^{+}$channel, and was thought to play several roles in cancer biology [28-31]. BK channels can promote growth and spreading of breast, prostate and gliomas tumor [32-35]. Some studies found that BK channels do not participate in glioma cell division [36] and genetic knock-down of BKa assist osteosarcoma development [37]. So the role of BK channel in human tumor may play a very complex one. In the above study, the researchers identified the $K C N M A 1$ generally acted as oncogene. However, in this study we found the KCNMA1 was down-regulated in the tumor tissues due to the methylation of promoter and played a tumor suppressor role. This finding uncovered the possible new mechanism that KCNMA1 was involved in carcinogenesis.

\section{Conclusion}

In conclusion, we have identified a novel tumor suppressive gene, KCNMA1, which is frequently inactivated in gastric cancer because of promoter methylation. KCNMA1 exerts a tumor suppressive function by regulating the PTK2 expression to activate the PI3K-AKT pathway. In addition, promoter hypermethylation of KCNMA1may serve as a potential prognostic biomarker in patients with gastric cancer.

\section{Additional files}

Additional file 1: Table S1. Clinical characteristics of 12 gastric cancer cases selected in microarray analysis. Table S2. Sequences of primers used in RT-PCR and MSP assay. (PDF $113 \mathrm{~kb}$ )

Additional file 2: Supplementary materials. (PDF $82 \mathrm{~kb}$ )

Additional file 3: Supplementary figure. (PDF $387 \mathrm{~kb}$ )

\section{Abbreviations}

BSP: Bisulphite sequencing; FAK: Focal adhesion kinase; GC: Gastric cancer; KCNMA1: Potassium channel, calcium activated large conductance subfamily $\mathrm{M}$ alpha, member 1; MSP: Methylation-specific PCR

\section{Acknowledgments}

This study was partly supported by National Natural Science Foundation of China (81473049, 81230068, and 81302490), Jiangsu Provincial Science and Technology Innovation Team, Jiangsu Provincial Postdoctoral Science Foundation funded project (1501081C), China Postdoctoral Science Foundation funded project (2015 M580449), Collaborative Innovation Center For Cancer Personalized Medicine, and the Priority Academic Program Development of Jiangsu Higher Education Institutions (Public Health and Preventive Medicine).

\section{Availability of data and materials \\ Yes}

\section{Authors' contributions}

$Z Z, W M, G W, M G, L H$, and $H Q$ designed and performed the research. $L Y, C H$, ZQ, QF, and TG collected data. DM, MG, and GY analyzed and interpreted data. DM and GY performed statistical analysis. MG, LH, and HQ wrote the draft manuscript. All authors contributed to the writing and reviewing of the manuscript, and approved the final manuscript for submission.

\section{Competing interests}

The authors declare that they have no competing interests.

\section{Consent for publication}

Yes 


\section{Ethics approval and consent to participate}

The research was approved by the Ethics Committee of Nanjing Medical University.

\section{Author details}

'Department of Environmental Genomics, Jiangsu Key Laboratory of Cancer Biomarkers, Prevention and Treatment, Cancer Center, Nanjing Medical University, Nanjing, China. ${ }^{2}$ Department of Genetic Toxicology, The Key Laboratory of Modern Toxicology of Ministry of Education, School of Public Health, Nanjing Medical University, Nanjing, China. ${ }^{3}$ Department of General Surgery, Yixing Tumor Hospital, Yixing, China. ${ }^{4}$ Department of General Surgery, The Second Affiliated Hospital of Nanjing Medical University, Nanjing, China. ${ }^{5}$ Core Laboratory, Nantong Tumor Hospital, Nantong, China. ${ }^{6}$ Department of General Surgery, Huai-An First People's Hospital Affiliated to Nanjing Medical University, Huai-An, China. ${ }^{7}$ Department of Environmental Genomics, School of Public Health, Nanjing Medical University, 101 Longmian AvenueJiangning District, Nanjing 211166, China.

Received: 23 July 2016 Accepted: 5 February 2017

Published online: 23 February 2017

\section{References}

1. Jemal A, Bray F, Center MM, Ferlay J, Ward E, Forman D. Global cancer statistics. CA Cancer J Clin. 2011;61:69-90.

2. Camargo MC, Kim WH, Chiaravalli AM, Kim KM, Corvalan AH, Matsuo K, Yu J, Sung JJ, Herrera-Goepfert R, Meneses-Gonzalez F, et al. Improved survival of gastric cancer with tumour Epstein-Barr virus positivity: an international pooled analysis. Gut. 2014;63:236-43.

3. Choi IS, Wu TT. Epigenetic alterations in gastric carcinogenesis. Cell Res. 2005:15:247-54.

4. Wang K, Liang Q, Li X, Tsoi H, Zhang J, Wang H, Go MY, Chiu PW, Ng EK, Sung JJ, YU J. MDGA2 is a novel tumour suppressor cooperating with DMAP1 in gastric cancer and is associated with disease outcome. Gut. 2016; 65:1619-31

5. Yu J, Cheng YY, Tao Q, Cheung KF, Lam CN, Geng H, Tian LW, Wong YP, Tong $\mathrm{JH}$, Ying $\mathrm{JM}$, et al. Methylation of protocadherin 10, a novel tumor suppressor, is associated with poor prognosis in patients with gastric cancer. Gastroenterology. 2009;136:640-51. e641.

6. Marabita F, Almgren M, Lindholm ME, Ruhrmann S, Fagerstrom-Billai F, Jagodic M, Sundberg CJ, Ekstrom TJ, Teschendorff AE, Tegner J, GomezCabrero D. An evaluation of analysis pipelines for DNA methylation profiling using the Illumina HumanMethylation450 BeadChip platform. Epigenetics. 2013;8:333-46.

7. Ouadid-Ahidouch $\mathrm{H}$, Ahidouch A. K+ channel expression in human breast cancer cells: involvement in cell cycle regulation and carcinogenesis. J Membr Biol. 2008;221:1-6.

8. Marrion NV, Tavalin SJ. Selective activation of Ca2 + -activated K+ channels by co-localized Ca2+ channels in hippocampal neurons. Nature. 1998;395:900-5.

9. Sah P, Faber ES. Channels underlying neuronal calcium-activated potassium currents. Prog Neurobiol. 2002:66:345-53.

10. Mizuno N, Yoshitomi H, Ishida H, Kuromi H, Kawaki J, Seino Y, Seino S. Altered bcl-2 and bax expression and intracellular $\mathrm{Ca} 2+$ signaling in apoptosis of pancreatic cells and the impairment of glucose-induced insulin secretion. Endocrinology. 1998;139:1429-39.

11. Li W, Ouyang Z, Zhang Q, Wang L, Shen Y, Wu X, Gu Y, Shu Y, Yu B, Sun Y, Xu Q. SBF-1 exerts strong anticervical cancer effect through inducing endoplasmic reticulum stress-associated cell death via targeting sarco/ endoplasmic reticulum Ca(2+)-ATPase 2. Cell Death Dis. 2014;5:e1581.

12. Mitra SK, Schlaepfer DD. Integrin-regulated FAK-Src signaling in normal and cancer cells. Curr Opin Cell Biol. 2006;18:516-23.

13. Zhao J, Guan JL. Signal transduction by focal adhesion kinase in cancer. Cancer Metastasis Rev. 2009;28:35-49.

14. Benjamini Y, Drai D, Elmer G, Kafkafi N, Golani I. Controlling the false discovery rate in behavior genetics research. Behav Brain Res. 2001;125:279-84.

15. Xu L, Li X, Chu ES, Zhao G, Go MY, Tao Q, Jin H, Zeng Z, Sung JJ, Yu J. Epigenetic inactivation of BCL6B, a novel functional tumour suppressor for gastric cancer, is associated with poor survival. Gut. 2012;61:977-85.

16. Wang S, Cheng Y, Du W, Lu L, Zhou L, Wang H, Kang W, Li X, Tao Q, Sung $\mathrm{JJ}, \mathrm{Yu}$ J. Zinc-finger protein 545 is a novel tumour suppressor that acts by inhibiting ribosomal RNA transcription in gastric cancer. Gut. 2013; 62:833-41.
17. Tomita H, Takaishi S, Menheniott TR, Yang X, Shibata W, Jin G, Betz KS, Kawakami K, Minamoto T, Tomasetto $C$, et al. Inhibition of gastric carcinogenesis by the hormone gastrin is mediated by suppression of TFF1 epigenetic silencing. Gastroenterology. 2011;140:879-91.

18. McLean GW, Komiyama NH, Serrels B, Asano H, Reynolds L, Conti F, Hodivala-Dilke K, Metzger D, Chambon P, Grant SG, Frame MC. Specific deletion of focal adhesion kinase suppresses tumor formation and blocks malignant progression. Genes Dev. 2004;18:2998-3003.

19. Shibue T, Weinberg RA. Integrin beta1-focal adhesion kinase signaling directs the proliferation of metastatic cancer cells disseminated in the lungs. Proc Natl Acad Sci U S A. 2009;106:10290-5.

20. Tavora B, Batista S, Reynolds LE, Jadeja S, Robinson S, Kostourou V, Hart I, Fruttiger M, Parsons M, Hodivala-Dilke KM. Endothelial FAK is required for tumour angiogenesis. EMBO Mol Med. 2010;2:516-28.

21. Sulzmaier FJ, Jean C, Schlaepfer DD. FAK in cancer: mechanistic findings and clinical applications. Nat Rev Cancer. 2014;14:598-610.

22. Jean C, Chen XL, Nam JO, Tancioni I, Uryu S, Lawson C, Ward KK, Walsh CT, Miller NL, Ghassemian M, et al. Inhibition of endothelial FAK activity prevents tumor metastasis by enhancing barrier function. J Cell Biol. 2014;204:247-63.

23. Cabrita MA, Jones LM, Quizi JL, Sabourin LA, McKay BC, Addison CL. Focal adhesion kinase inhibitors are potent anti-angiogenic agents. Mol Oncol. 2011:5:517-26.

24. Konstantinidou G, Ramadori G, Torti F, Kangasniemi K, Ramirez RE, Cai Y, Behrens C, Dellinger MT, Brekken RA, Wistuba II, et al. RHOA-FAK is a required signaling axis for the maintenance of KRAS-driven lung adenocarcinomas. Cancer Discov. 2013:3:444-57.

25. Pylayeva Y, Gillen KM, Gerald W, Beggs HE, Reichardt LF, Giancotti FG. Ras- and PI3K-dependent breast tumorigenesis in mice and humans requires focal adhesion kinase signaling. J Clin Invest. 2009;119:252-66.

26. Chen JS, Huang XH, Wang Q, Huang JQ, Zhang $\amalg$, Chen XL, Lei J, Cheng ZX. Sonic hedgehog signaling pathway induces cell migration and invasion through focal adhesion kinase/AKT signaling-mediated activation of matrix metalloproteinase (MMP)-2 and MMP-9 in liver cancer. Carcinogenesis. 2013; 34:10-9.

27. Vaithianathan T, Bukiya A, Liu J, Liu P, Asuncion-Chin M, Fan Z, Dopico A. Direct regulation of BK channels by phosphatidylinositol 4,5-bisphosphate as a novel signaling pathway. J Gen Physiol. 2008;132:13-28.

28. Lang F, Foller M, Lang KS, Lang PA, Ritter M, Gulbins E, Vereninov A, Huber SM. Ion channels in cell proliferation and apoptotic cell death. J Membr Biol. 2005;205:147-57.

29. MacFarlane $\mathrm{SN}$, Sontheimer $\mathrm{H}$. Changes in ion channel expression accompany cell cycle progression of spinal cord astrocytes. Glia. 2000;30:39-48.

30. Weaver AK, Liu X, Sontheimer H. Role for calcium-activated potassium channels (BK) in growth control of human malignant glioma cells. J Neurosci Res. 2004;78:224-34.

31. Pancrazio JJ, Tabbara IA, Kim YI. Voltage-activated K+ conductance and cell proliferation in small-cell lung cancer. Anticancer Res. 1993;13:1231-4.

32. Bloch M, Ousingsawat J, Simon R, Schraml P, Gasser TC, Mihatsch MJ, Kunzelmann K, Bubendorf L. KCNMA1 gene amplification promotes tumor cell proliferation in human prostate cancer. Oncogene. 2007;26:2525-34.

33. Khaitan D, Sankpal UT, Weksler B, Meister EA, Romero IA, Couraud PO, Ningaraj NS. Role of KCNMA1 gene in breast cancer invasion and metastasis to brain. BMC Cancer. 2009;9:258

34. Bury M, Girault A, Megalizzi V, Spiegl-Kreinecker S, Mathieu V, Berger W, Evidente A, Kornienko A, Gailly P, Vandier C, Kiss R. Ophiobolin A induces paraptosis-like cell death in human glioblastoma cells by decreasing BKCa channel activity. Cell Death Dis. 2013;4:e561.

35. Basrai D, Kraft R, Bollensdorff C, Liebmann L, Benndorf K, Patt S. BK channel blockers inhibit potassium-induced proliferation of human astrocytoma cells. Neuroreport. 2002:13:403-7.

36. Abdullaev IF, Rudkouskaya A, Mongin AA, Kuo YH. Calcium-activated potassium channels BK and IK1 are functionally expressed in human gliomas but do not regulate cell proliferation. PLoS One. 2010;5:e12304.

37. Cambien B, Rezzonico R, Vitale S, Rouzaire-Dubois B, Dubois JM, Barthel R, Karimdjee BS, Mograbi B, Schmid-Alliana A, Schmid-Antomarchi H. Silencing of hSlo potassium channels in human osteosarcoma cells promotes tumorigenesis. Int J Cancer. 2008;123:365-71. 